\title{
Adaptive Modulation and Code Strategy to Reduce Energy Consumption in Elastic Optical Network
}

\author{
S.Y.M. Bandiri, T.C. Pimenta and D.H. Spadoti \\ Institute of Systems Engineering and Information Technology, Federal University of Itajubá, Brazil \\ Emails: bandiri@unifei.edu.br, tales@unifei.edu.br, spadoti@unifei.edu.br
}

\begin{abstract}
In this paper, the novel Adaptive Modulation and Code (AMC) algorithm aims to reduce energy consumption in elastic optical network is developed. The proposed AMC algorithm adaptively allocates both, the appropriate modulation and the forward error correction (FEC), according to the actual physical distance and the optical signal to noise ratio (OSNR) of the lightpath, respectively. The algorithm compares the previous energy consumption with the actual aiming to select the lowest. The obtained results have been compared with the case when shortest path (SP) and minimum hops (MH) algorithms are employed. Simulations outcomes highlight that energy consumption decreases when AMC algorithm is adopted in comparison with SP or MH ones. The energy consumption increases proportionally with the physical lightpath distance. Finally, the blocking probability decreases whereas AMC is utilized.
\end{abstract}

Index Terms - Adaptive FEC, Adaptive Modulation, Blocking Probability, Energy Consumption.

\section{INTRODUCTION}

The rapid growth in the use of information and communications technologies (ICT), led to the increase of the global energy consumption [1]-[3]. Recent estimates depict out that the power consumption has doubled around the world in the latest years and ICT accounted approximately for $4 \%$ [2]. According to Rouzic [4], reducing the energy consumption is becoming a key environmental, social and political issues. To this end, several researches have deployed various techniques and algorithms aiming to reduce energy consumption [4]-[9]. However, energy consumption reduction continues being a challenge. Thus, it is imperative to deploy energetic efficiency solutions while the quality of service (QoS) and quality of transmission (QoT) criteria are remained. 
In traditional wavelength division multiplexing (WDM), the spectrum is shared into fixed bandwidth by imposing rigid transmission rates at each wavelength transmitting high bit rates with channels operating between 40Gbps and 100Gbps [10]. Consequently, the spectrum efficiency utilization is reduced, often leading to under-utilization or over-provisioning of resources due to the different granularities of demand generated in customer layers. Further, WDM technology does not solve some critical limitations of the physical layer which cannot be ignored, such as: channel density spacing, different nonlinear effects (Raman and Brillouin scattering, four-wave mixing (FWM), crossphase modulation (XPM), self-phase modulation (SPM)), among others [11]. Thus, WDM optical network has the poor resource utilization and it is mandatory to deploy new paradigms making the optical network more flexible.

Aiming to attend this network flexibility, elastic optical network (EON) emerges as a promising solution for the future of high-speed optical transmission and a potential candidate to overcome WDM limitations [12]. This scheme has been projected as a way to assign bandwidth to optical path (referred as lightpath), based on their needed transmission rates [13]. When a new connection is solicited, EON considers the most effective implementation of modulation format, forward error correction (FEC) and spectrum, yielding to the lowest-cost solution. This decision relies on adaptive behavior that adjusts itself based on actual link conditions and control plane impairments knowledge whereas it still considering margins for network aging [10].

Reference [14] has considered the transmission distance as the unique QoT factor of interest. The applicable modulation level with acceptable QoT was determined as a function of the transmission distance. However, the longest transmission distance depends on some factors, including fiber losses, OSNR limits, among others, and it must be evaluated accurately for effective distance-adaptive schemes. For the spectrum request's allocation, only one modulation scheme is adopted in their paper.

In [15], a new impairment-aware routing spectrum assignment (RSA) algorithm in flexible orthogonal frequency-division multiplexing (OFDM) based networks was recently studied, but only binary phase shift keying (BPSK) was used as modulation. The question of how to improve the spectrum utilization under the constraint of physical impairments remains to be the important open issue in flexible optical networks.

OFDM has receiving more attention lastly due to the multiple characteristics to support high-speed optical transmission technology [15]. OFDM systems are based on multi-carrier transmission enabling high speed data stream, which are split into multiple parallel low-speed data channels. In OFDM, link adaptation is possible according to channel conditions and custom bandwidth requirement driving to enhance higher spectrum efficiency and power consumption reduction. Based on these flexibility and scalability characteristics of optical OFDM, a novel Elastic Optical Network (EON) was proposed 
[12]. The elastic optical network breaks the fixed-grid wavelength-allocation limitation of WDM by adjusting network parameters according to the channel conditions. EON supports multiple data rate sub-wavelength or super-wavelength, achieving, thus, high spectrum efficiency [10], [12], [13].

In EON, optical orthogonal frequency division multiplexing (O-OFDM) splits a high data rate stream onto several lower symbol rate subcarriers [10]. Consequently, they are turned more susceptible to physical layer impairments. The optical signal to noise ratio (OSNR) of a high data rate optical signal would degrade significantly over long transmission distances [16]. Forward error correction (FEC) coding techniques is an optimal candidate to compensate this degradation through a high error correction performance with low hardware investment. This is made possible by adding redundant overhead bits appropriately to the information bits before modulation and transmission. The FEC overhead can be changed without disrupting the traffic, if the decoder knows exactly where in the bit stream this change is taking place. There is a trade-off between the improvement in the net coding gain (NCG) obtained through the FEC and the increase in its coding overhead $(\mathrm{OH})$ for a system using FEC [16], [17].

In traditional system when a demand arrives to an optical network, a fixed FEC allocation is adopted by choosing the FEC with high NCG with the poorest OSNR in the whole network. This is wasteful when a lightpath requires FEC type with lower $\mathrm{OH}$ in order to support the desired bit error rate (BER). For this reason, we considered beyond of adaptive modulation, an adaptive FEC allocation strategy.

As a main contribution of this paper, the novel strategy, based on Adaptive Modulation and Coding (AMC) is proposed, yielding to reduce the power consumption in an EON. In the proposed algorithm, the modulation format and the type of FEC code vary as function of the lightpath distance and the lightpath OSNR, respectively. This proposed AMC algorithm ensures both the quality of service (QoS) and quality of-transmission (QoT) criteria.

The remaining of the paper is organized as follows: the model of power consumption over optical network is described in Section II. In Section III, the novel algorithm AMC is presented and the results are discussed in Section IV. Finally, the paper is concluded in Section V.

\section{BUILDING BLOCKS OF OFDM SYSTEMS FOR OPTICAL LIGHTPATH AND POWER MODEL}

In this proposal study, a generic building block diagram of an optical orthogonal frequency division multiplexing (O-OFDM) system is considered. The O-OFDM plays an important role by permitting an elastic bandwidth transmission. For every connection request, the transmission rate can be adjusted according to the traffic by expanding or contracting the bandwidth of an optical path. The block 
diagram of Fig.l represents the set of components, such as: transmitter, optical fiber spans with optical amplifiers, bandwidth variable optical cross-connects (BV-OXCs), and receiver. This diagram block is not stationary, instead it varies according to systems specifications, bit rate, code rate OSNR, and distance between nodes.

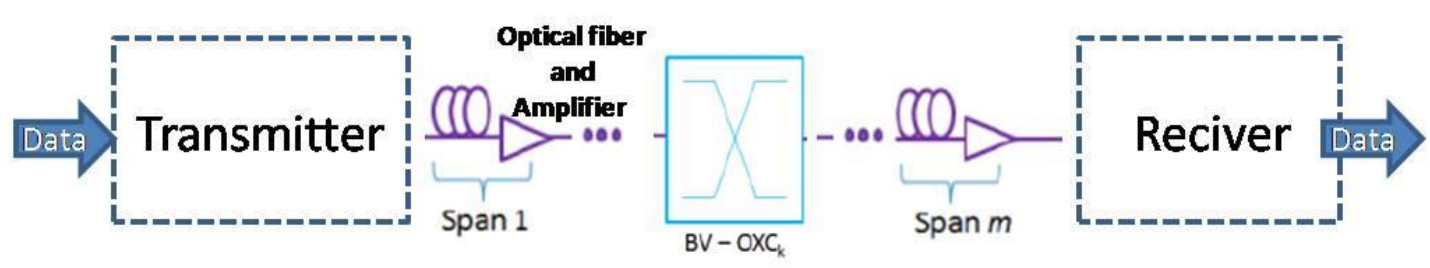

Fig. 1. Block diagram of end-to-end lightpath OFDM-based transmission system

For a determinate lightpath O-OFDM-based, the total energy consumption, $E_{\text {optical }, i}$ can be computed as following [18]:

$$
E_{\text {optical }, i}=E_{T X i}+E_{T R i}+\sum_{q=1}^{Q} E_{E D F A(q) i}+\sum_{r=1}^{R} E_{B V-O X C(r) i}
$$

In (1), $E_{T X i}$ and $E_{R X i}$ are the energy consumption of the transmitter and the receiver, respectively. $E_{E D F A(q) i}$ is the energy consumption of the $q_{t h}$ Erbium Doped Fiber Amplifiers (EDFAs) in the optical routes (booster, in-line, in the BV-OXCs and at the receiver), $E_{B V-O X C(r) i}$ is the energy consumption of each $r_{t h} B V-O X C(r) i$. These energy consumptions are considered per information bit. In this paper, Binary Phase Shift Keying (BPSK), Quadrature-PSK (QPSK), 8-PSK and M-ary Quadrature Amplitude Modulation (M-QAM), where $M=16,32$ or 64 symbols have been used. Every modulation provides different spectral efficiency expressed in bit/s/Hz. The complementary formulation and tables with value of each parameters and variables are highlight detailed in appendix A, B and C.

Traditional network design is based on a worst-case approach. Therefore, any path out of the worst case, has unused margins in terms of OSNR or transmission impairment. O-OFDM allows distanceadaptive bit-rate and spectrum allocation to conserve spectral resources for shorter paths. Hence, the analytical formula (distance reach versus bit rate) for coherent systems which includes physical layer constraints as fiber loss, $\mathrm{OSNR}_{\text {limits }}$, nonlinear effects like cross phase modulation (XPM), four wave mixing (FWM)and arbitrary neighboring WDM channels is used [19]:

$$
D_{\max }=\frac{1}{2}\left[-804.3 \times \ln \left(B_{r}\right)+5584.6\right]
$$

In (2), $D_{\max }$ denotes the maximum distance transmission (MDT) reach in (Km), $B_{r}$ denotes the bit rate in Gbps. Table I summarizes the maximum distance transmission reaches for every modulation Brazilian Microwave and Optoelectronics Society-SBMO received 09 Oct 2017; for review 13 Oct 2017; accepted 01 Feb 2018 Brazilian Society of Electromagnetism-SBMag 
format and the correspondent subcarrier for a single sub-channel with $12.5 \mathrm{GHz}$ of bandwidth.

TABLE I. MAXIMUM DISTANCE TRANSMISSION OF AN O-OFDM FOR DIFFERENT MODULATION FORMATS

\begin{tabular}{ccc}
\hline Modulation Format & Subcarrier Capacity (Gbps) & MDT $(\mathbf{K m})$ \\
\hline BPSK & 12.5 & 1776 \\
QPSK & 25 & 1219 \\
8PSK & 37.5 & 893 \\
16QAM & 50 & 662 \\
32QAM & 62.5 & 483 \\
64QAM & 75 & 336 \\
\hline
\end{tabular}

As we have mentioned previously, the FEC type is selected according to the actual lightpath OSNR. To efficiently use adaptive FEC benefit, by reducing the overhead, the frequency slot, all the three FEC types should be used [16]. From Table II, we appreciate that the first generation of FEC, i.e., RS $(255,239)$ requires the lowest net coding gain $(\mathrm{NCG})$ and the lowest overhead $(\mathrm{OH})$ while achieving the highest OSNR limit. In contrast, the third FEC type generation, i.e., LDPC $(4161,3431,0.825)$ has the lowest OSNR limit while shows the lowest OH and NCG. The second generation of FEC, i.e., RS $(255,239) / \mathrm{BCH}(1023,963)$ carries out an intermediate performance among the previous generations mentioned. In general, an FEC type with better performance has lowest OSNR limit, highest NCG and $\mathrm{OH}$.

The three types of FEC code considerate in this work are summarized in the Table II [16].

TABLE II : Generations OF FEC CODES

\begin{tabular}{cccc}
\hline Generation & Type of FEC Code & OSNR(dB) Limit & Overhead (\%) \\
\hline FEC1 & RS $(255,239)$ & 14.5 & 6.69 \\
FEC2 & RS $(255,239) /$ & 12.6 & 13.34 \\
& BCH $(1023,963)$ & & \\
FEC3 & LDPC $(4161,3431,0.825)$ & 9.1 & 21.20 \\
\hline
\end{tabular}

The optical signal to-noise ratio for every lightpath can be computed by the following equation [16]:

$$
\begin{aligned}
& P_{A s e}(d B m)=-58(d B m)+G(d B)+N F_{G}(d B) \\
& P_{A s e}(m W)=\sum_{i=1}^{f} P_{A s e}^{i}=\sum_{i=1}^{f} 10^{\left(P_{A s e}^{i}(d B m) / 10\right)} \\
& O S N R_{S}=P_{\text {out }} / P_{\text {Ase }}=1 / P_{\text {Ase }}(m W) \\
& \frac{1}{O S N R_{\text {Total }}}=\frac{1}{\sum_{s} \varepsilon S O S N R_{s}}
\end{aligned}
$$

Brazilian Microwave and Optoelectronics Society-SBMO received 09 Oct 2017; for review 13 Oct 2017; accepted 01 Feb 2018 
In (3), $P_{\text {Ase }}$ is the power of the ASE noise of an optical amplifier, $G$ is the optical amplification gain and $N F_{\mathrm{G}}$ is the corresponding noise figure value when the amplification gain is G. In (5), $P_{\text {out }}$ $\left(P_{\text {out }}=1 \mathrm{~mW}\right)$ denotes the output power of a lightpath. In $(4), f(f=1,2,3, \ldots, N)$ is the total number of EDFAs amplifiers. In (6), the set of links traversed by the lightpath is denoted by $S$ with the corresponding OSNRs; $O S N R_{\text {Total }}$ is the OSNR of a lightpath that traverses multiple links and $\in$ : is a mathematic symbol meaning "set membership". The above equations can be explained individually as follows: (3) calculates the power of the ASE noise associated of an optical amplifier in (dBm); (4) computes the finds the accumulated ASE noise on a fiber link which contains $n$ EDFAs; (5) calculates the OSNR of the fiber link and the OSNR of a lightpath that traverses multiple links is computed in (6). When a connection is solicited, the lightpath OSNR is computed through (3), (4), (5) and (6). After that, the appropriate type of FEC to be used is selected according to the OSNR limit.

\section{AMC-RSA ALGORITHM}

The proposed AMC algorithm aims to reduce the energy consumption in EON by adaptively allocating the appropriate modulation and FEC according to the actual physical distance and the OSNR of a lightpath respectively. The algorithm also uses "backup mode" (we consider that in this mode, the AMC algorithm compares the energy consumption of the actual lightpath with the previous) to select the lowest energy consumption for every incoming connection request. The main idea of AMC algorithm is explained as following: when $n(n=1,2,3, \ldots . N)$ connection requests arrive to the EON:

- Two nodes are randomly selected one as the source node and the other as the destination (all nodes are equiprobable i.e. they have the same probability to be selected)

- The Yen algorithm [20] is executed in order to choose $k(k=1,2,3, \ldots . . K)$ shortest paths between the selected nodes.

- The OSNR and the physical distance of the $k$ shortest paths are computed.

- The proper FEC code type is assigned to each lightpath according to their respective OSNR. If the OSNR value of the lightpath is less than $\mathrm{OSNR}_{\text {limit }}=9.1 \mathrm{~dB}$, then there is no need to use any FEC type for this case.

- The correspondent modulation format is assigned to each lightpaths.

- The energy consumption $\left(E_{1}, E_{2}, E_{3}, \ldots E_{K}\right)$ of the $k$ shortest paths is calculated and the lowest is chosen.

- The above processes are repeated for all $N$ connection requests.

- If no modulation and FEC type are found even after adjusting the baud rate, the corresponding connection request is blocked. The connection request is blocked if all the $k$ 
shortest chosen paths are blocked. In other words, if no modulation is allocated to the $k$ shortest paths.

- If a connection is blocked, the next connection is attended following the above process until the accomplishment of the $N$ incoming requests.

The following considerations have been performed: the maximum transmission distance reaches by each modulation is calculated through (2) and recorded in $d M p s k(\mathrm{M}=2,4,8$ which correspond to BPSK, QPSK, 8PSK respectively) and dMqam (M = 16, 32, 64 corresponding to 16QAM, 32QAM, 64QAM respectively). In the algorithm, "Cost" is the physical distance among the source node and the destination node. $E_{K}$ is the power consumption of the $k_{t h}$ lightpath. Toward this end, the new AMC algorithm is introduced in the following: 


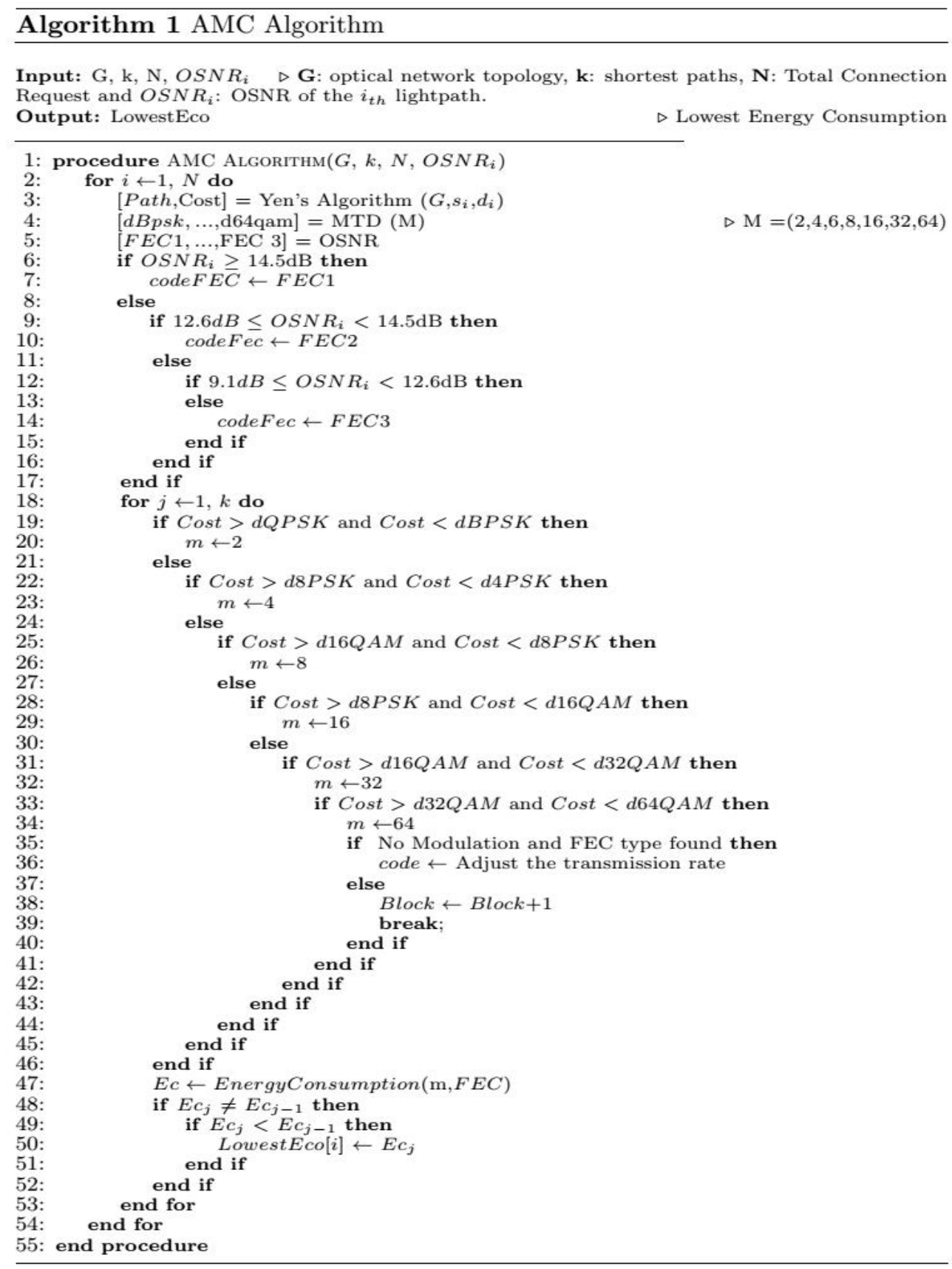

\section{R ESULTS AND DISCUSSION}

In order to evaluate the AMC algorithm performance, extensive simulations have been conducted through the US nationwide and Germany network topology and illustrated in Fig.2 and Fig.3 respectively. To create a dynamic scenario incoming connection requests, arrive to the OEN following Poisson process with an average arrival rate $\lambda$ and the duration time is exponentially distributed. The numbers of connection requests vary from 0 to 1000 . We consider all the possible node pairs on the network $(552=24 \times 23$ nodes pairs US nationwide network and 272=17x16 nodes pairs Germany network). All the intermediate nodes among source and destination will be considered as hops. In 
addition, to guarantee the same input conditions for the algorithm a random number generator with a constant seed is considered. The interval bit rate varies from $40 \mathrm{Gbps}$ to $250 \mathrm{Gbps}$.

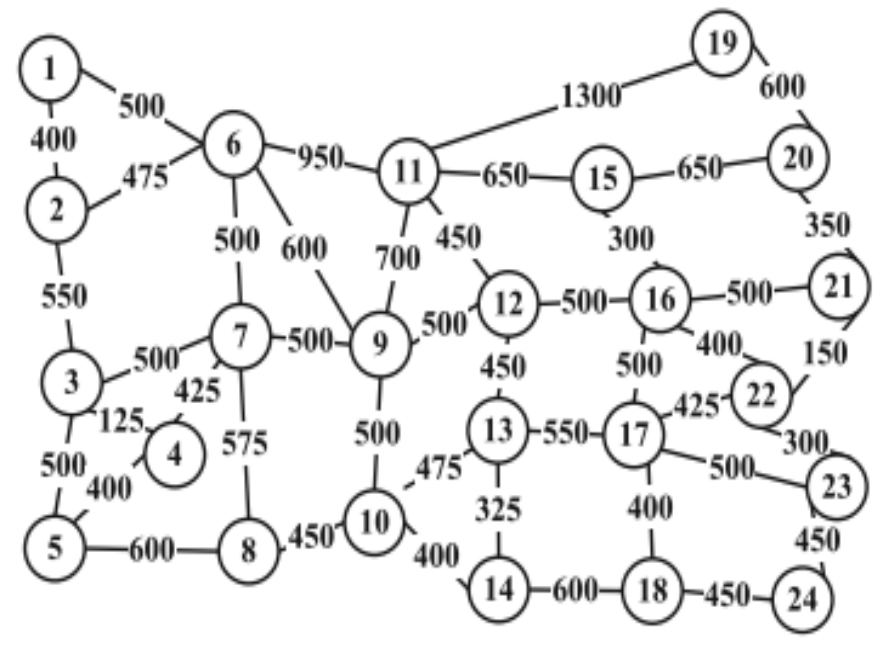

Fig. 2. US Nationwide network [11]

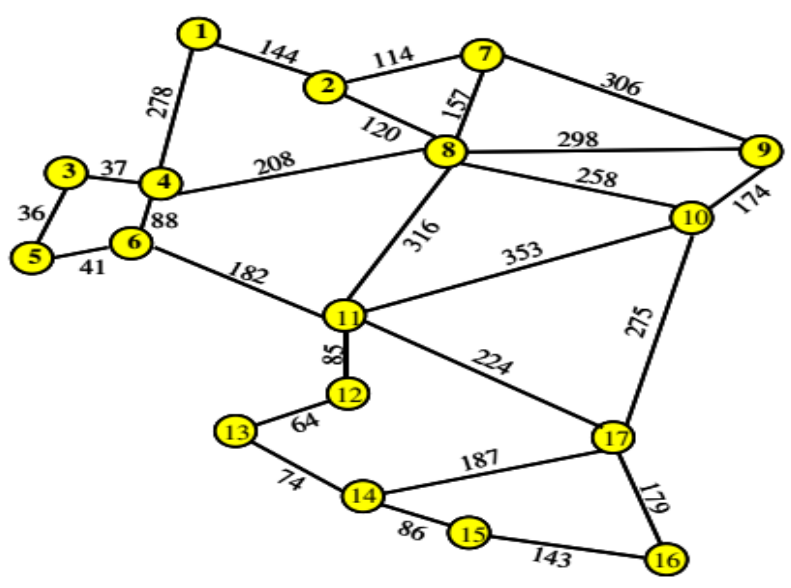

Fig. 3. Germany Network [21]

In first instance, we suppose that there is not connection blocked in the EON i.e. all incoming request connections are attended. The energy consumption is computed and results have been compared with the traditional shortest path (SP) and minimum hops (MH) algorithms respectively [22], [23]. Every algorithm will operate as resumed below:

- SP: employs fixed modulation and FEC for all incoming connection requests and uses Dijkstra algorithm to select the shortest path. Finally, the energy consumption for the shortest path is computed. 
- MH: selects the lightpath with minimum hops and adopts fixed modulation and codification for all incoming connection requests; Finally, the energy consumption of selected lightpath is calculated.

- AMC: executes Yen's algorithm in order to select $k$-shortest path [20]. After that, the proper FEC type and modulation format are allocated for every $k$ selected shortest path. Finally, the energy consumption is computed and lowest is adopted.

Fig. 4 and Fig. 5 show the energy consumption per information bit versus connection requests for the three different algorithms in US nationwide network and Germany network respectively. In this first case, we assume that all the connection request is attended. The simulations results highlight that the energy consumption increases proportionally with the growth of the connection requests for all the three algorithms in both networks. Furthermore, AMC reveals to the lowest energy consumption following by the $\mathrm{MH}$ algorithm and finally, the SP algorithm. The main reason is that the AMC algorithm selects the "best" modulation format and FEC type adaptively for the connection request according to the physical distance of the traversed link driving to use less spectrum-efficient modulation schemes and less FEC overhead. From general transmission theory, less spectrum efficient modulation schemes can function at a lower signal-to-noise ratio (SNR) for a given bit error rate (BER) and by inserting extra parity bits at the cost of lower effective throughput will increase the FEC's ability to correct errors and thus provide a so-called coding gain corresponding to increasing the SNR. Hence, less regeneration, amplification, and signal processing will be needed. As with the symbol rate, the digital electronics such as the FEC can also run at a lower rate, thus saving energy consumption. Additionally, AMC exploits the flexibility given by different transmission rates, which allows for a better adjustment of the transmission rate to the actual capacity requested.

The energy consumption in the Germany network is lower than in the US nationwide network. The main reason is that the US nationwide network is higher than the Germany network and the physical distance between two consecutive nodes are also higher than in the Germany network.

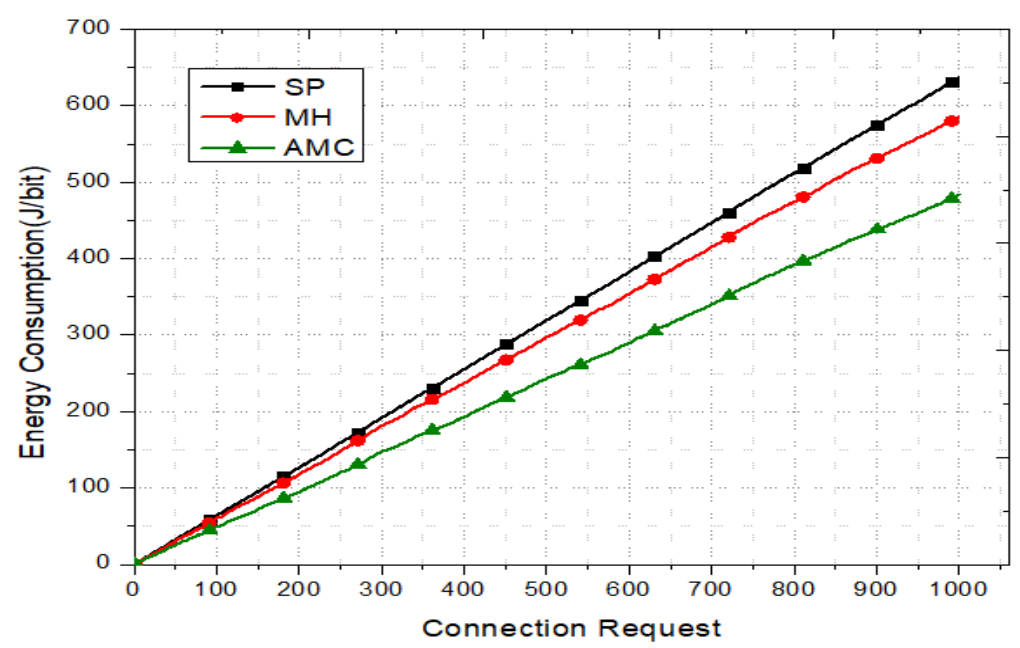

Brazilian Microwave and Optoelectronics Society-SBMO Brazilian Society of Electromagnetism-SBMag 
Fig. 4. Power consumption versus Connection Requests for SP, MH and AMC algorithms with US nationwide network.

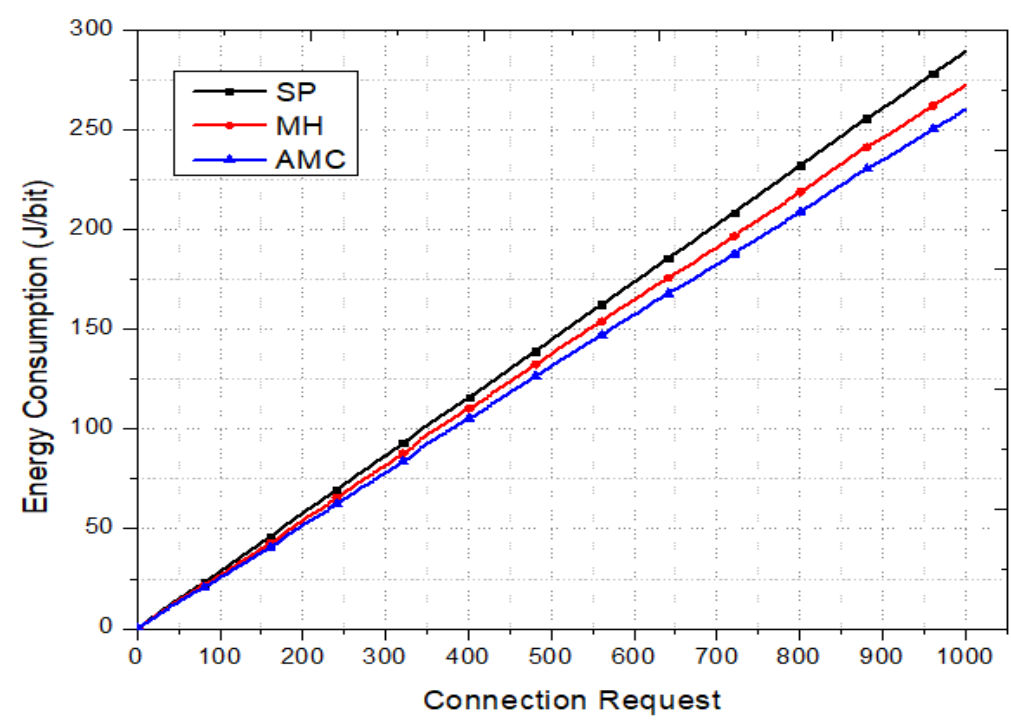

Fig. 5. Energy consumption versus Connection Requests for SP, MH and AMC algorithms with Germany network.

Table III and Fig.6 summarize the energy consumption in the EON by using the three algorithms SP, MH and AMC. Seven different lightpaths have been chosen and results show that in each lightpath, AMC algorithm outperforms MH and SP algorithm. For all the three algorithms, the energy consumption increases with the distance among the source and destination node. This can be explained by the fact that, the more long is the lightpath, more hops, EDFAs and BV-OXCs are traversed yielding to increment the energy consumption. The total energy consumption for the seven lightpaths is their arithmetic sum. Thus, the total energy consumption by using SP, MH and AMC algorithms are 3.963695J/bit, 2.188561J/bit and 1,991452J/bit respectively.

TABLE III: ENERgy CONSUMPTION FOR DIFFERENT LIGHTPATHS USING SP, MH AND AMC ALGORITHMS USING USA NATIONWIDE NETWORK.

\begin{tabular}{cccccc}
\hline \multicolumn{2}{c}{ LIGHTPATHS } & \multirow{2}{*}{ DISTANCE(KM) } & SP(J/bit) & MH(J/bit) & \multirow{2}{*}{ AMC(J/bit) } \\
\cline { 1 - 2 } SOURCE & DESTINATION & & & & \\
\hline 3 & 11 & 1700 & 0.652711 & 0.652711 & 0.600268 \\
14 & 16 & 1275 & 0.607760 & 0.336729 & 0.299270 \\
6 & 11 & 950 & 0.561130 & 0.260310 & 0,237830 \\
20 & 16 & 850 & 0.559452 & 0.258632 & 0,236343 \\
22 & 24 & 750 & 0.553639 & 0.252818 & 0.220343 \\
5 & 8 & 600 & 0.529485 & 0.228664 & 0.206189 \\
23 & 22 & 300 & 0.499518 & 0.198697 & 0.191205 \\
\hline
\end{tabular}




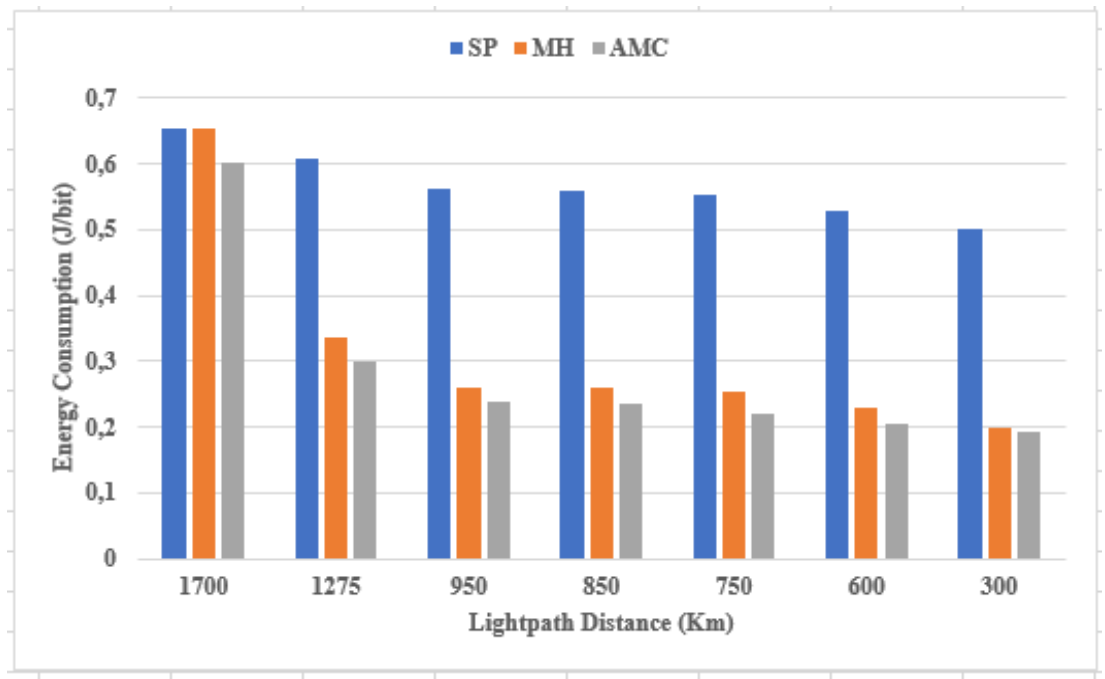

Fig. 6. Energy consumption versus Distance for SP, MH and AMC algorithms with USA nationwide network.

Now, the possibility to have connection blocking in the EON is included in the simulations. Incoming connection is blocked when the lightpath distance is greater than the MDT of BPSK or the maximum blocking allowed in the optical network is reached. The blocking probability can be defined as the ratio between the number of request connection failed to the total connection number that arrives in the EON.

Fig.7 and Fig.8 show the blocking probability in function of the network transmission rate. We assume that $N=100,500$ and 1000 connections requests (CR) have to be attended respectively. Both graphics reveal that the blocking probability increases proportionally as the transmission rate enhances. However, the blocking probability decreases by increasing the connection request. The blocking probability is high in USA network than in Germany network. This could be explained by the fact that in small network (as Germany network), the distance among two consecutives nodes is small than in large network (as US network) where the physical distance is more longer. 


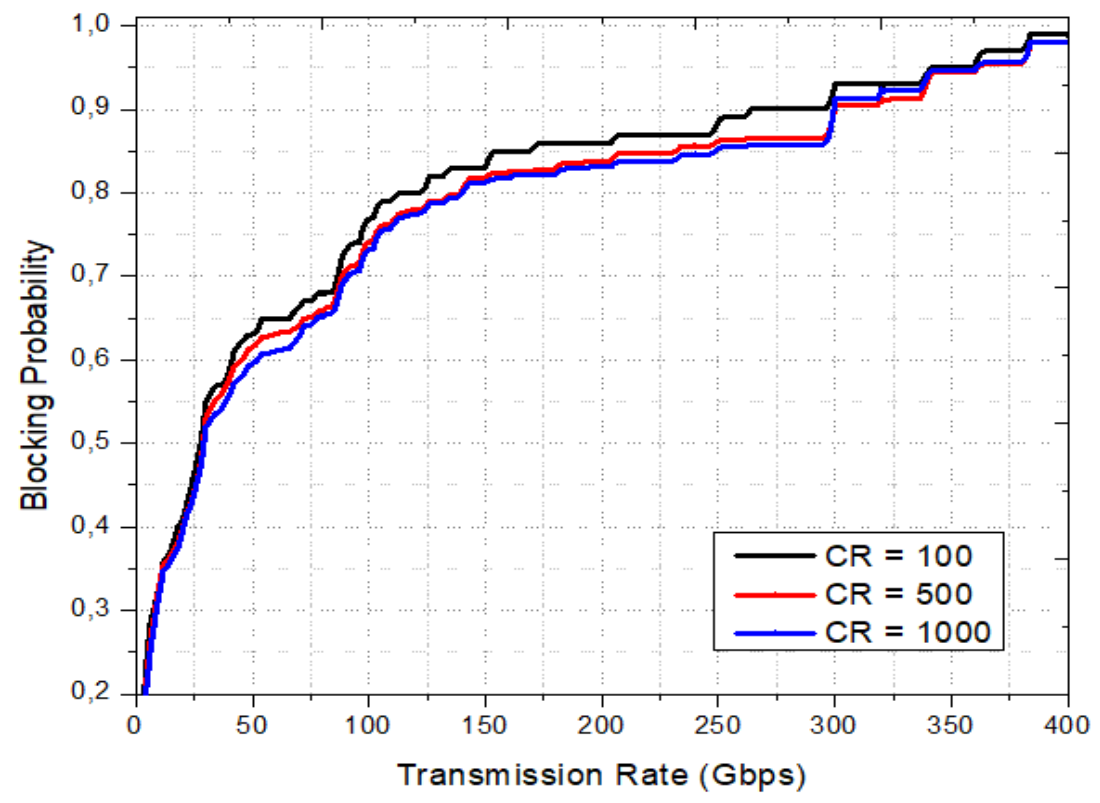

Fig. 7. Blocking Probability versus Transmission for connection request equal to 100; 500; 1000 using US nationwide network.

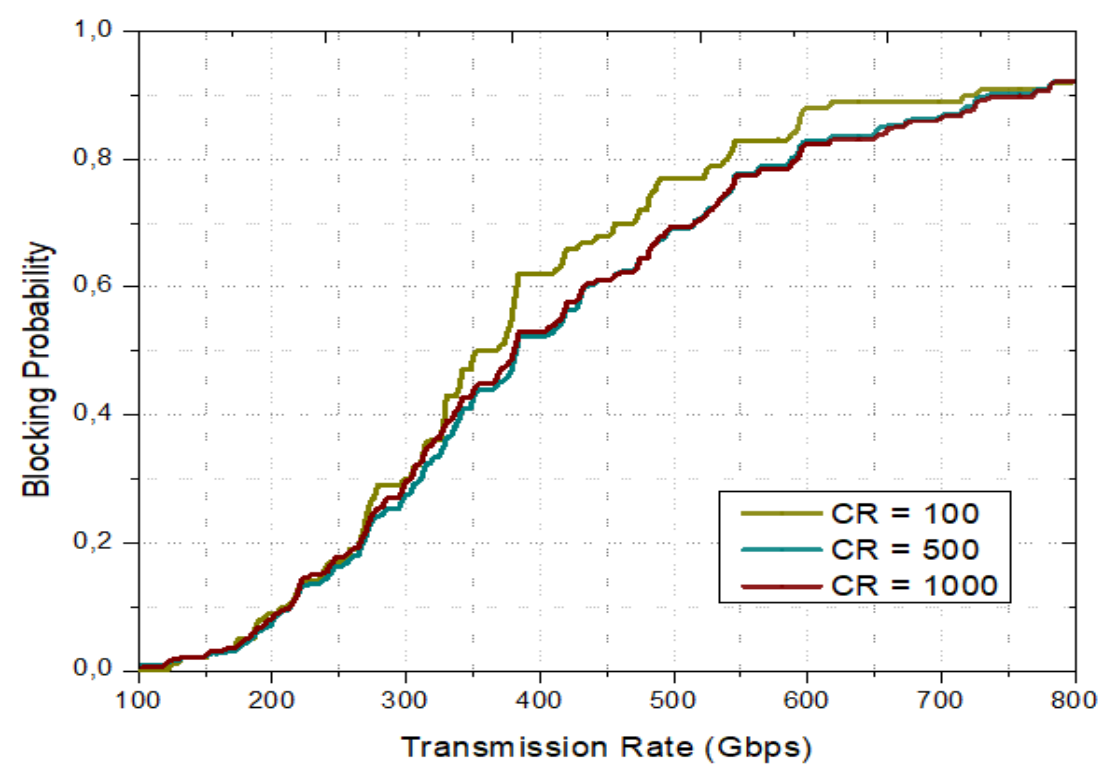

Fig. 8. Blocking Probability versus Transmission Rate for connection request equal to $100 ; 500 ; 1000$ using Germany network.

Finally, Fig.9 highlights the performance of AMC algorithm investigated in terms of blocking probability versus incoming connections requests. More again, AMC algorithm outperforms the others SP and MH algorithms. The AMC algorithm exploits Bandwidth Variable Transponders (BVTs) function that can tune their bit-rate and bandwidth dynamically with a trade-off between reach and capacity. Consequently, in this paper, the bit rate is adjusted (by decreasing) to the lower value within the defined range. This allows increasing the maximum distance reach by each modulation format, thus reducing the blocking probability. Furthermore, the connection request is 
blocked if, and only if, all the $k$-shortest paths selected are blocked in contrast in SP and MH algorithm, only one lightpath is selected

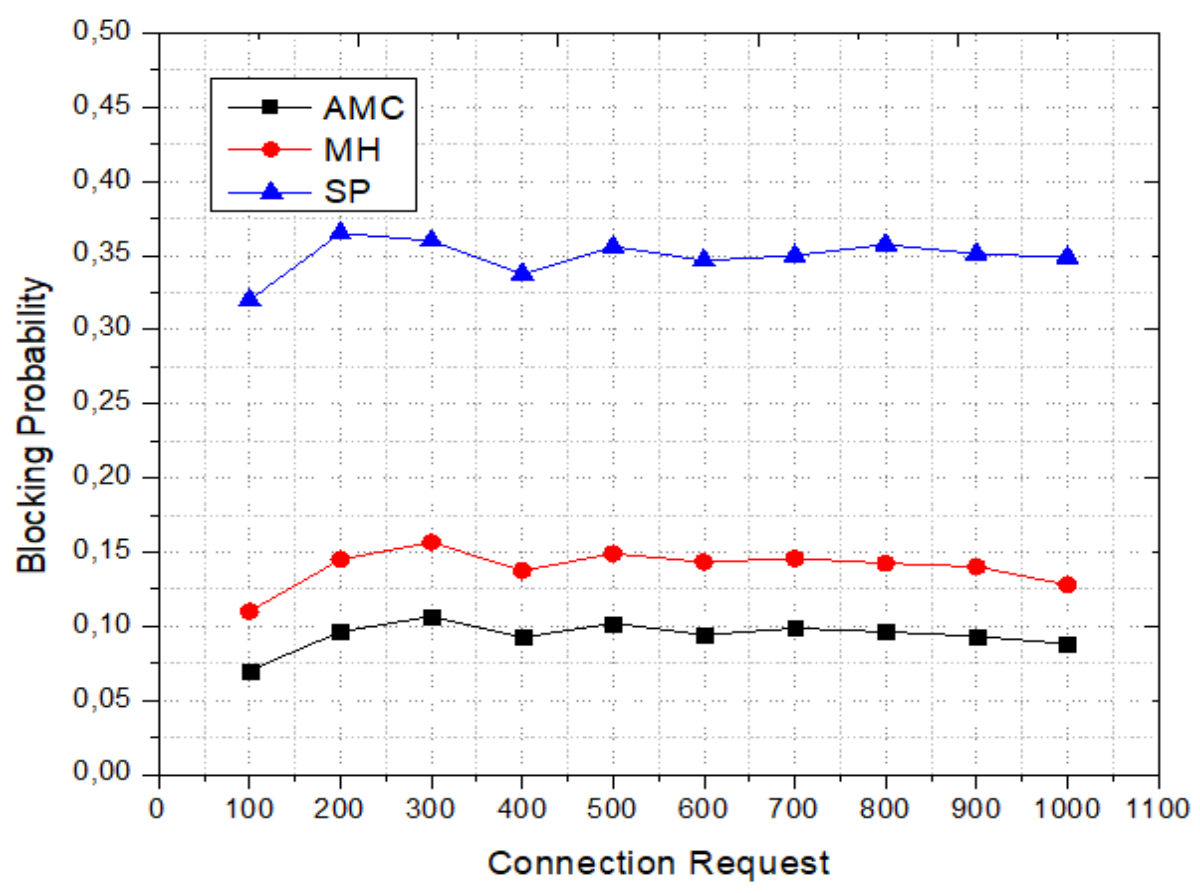

Fig. 9. Blocking probability as function of connections requests using US nationwide network.

Our proposed AMC is a good strategy to reduce energy consumption in EON and still ensures QoT and QoS requirement. The limitation of this proposal algorithm is the average run time which higher than MH and SP algorithms. And just for illustration, under the same conditions, (assuming 1000 connections requests, system without blocking connection) the average run time of AMC, MH and SP algorithms are $87.129 \mathrm{~s}, 34.351 \mathrm{~s}$ and $42.137 \mathrm{~s}$, respectively. The reason is that the time select $k$ shortest paths, compute their respective ONSR, find their physical distance and then allocate the appropriate FEC type, modulation format for each connection request is longer than the others algorithms. However, AMC algorithm is preferred since it outperforms MH and SP algorithms in the mains goal of this paper.

\section{CONCLUSION}

Through the novel AMC algorithm optical energy consumption is reduced in EONs. The energy consumption grows proportionally with the physical distance of the lightpath and the optical network size. The blocking probability increases proportionally with the transmission rate; however, it decreases when the connection request increase in the EON. The proposed algorithm also outperforms the analyzed SP and MH algorithms in terms of blocking probability. In future work, this novel AMC algorithm will be applied in order to compute energy efficiency in elastic optical network. 


\section{APPENDIX A: ENERGY CONSUMPTION CALCULATION}

$E_{\text {optical }, i}=E_{T X i}+E_{R X i}+\sum_{q=1}^{Q} E_{E D F A(q) i}$

$E_{T X i}$ is the energy Consumption at the transmitter, $E_{R X i}$ is the energy consumption at the receptor, $E_{E D F A(q) i}$ is the energy consumption for the EDFA amplifiers.

The transmitter power consumption can be computed as:

$$
E_{T X i}=\frac{R_{i}}{\eta_{E O}}\left(E_{F E C}+E_{P-S}+E_{D A C}+E_{M O D}\right)+\frac{\eta_{T} R_{i}}{2}+P_{C W}
$$

and the calculation of each variable is defined as follow:

$E_{F E C}$ is the encoding energy consumption which could be the LDPC or RS code.

$E_{L D P C}^{e n}(l k)=\frac{\left[(l-k)\left(k c_{g}-1\right) E_{o p}^{G}+l E_{o p}^{R}\right]}{k}$

$E_{L D P C}^{e n}$ is the energy Consumption of the LDPC code. $l, k$ are the code length and the numbers of information bit per code blocks respectively. $\mathrm{C}_{\mathrm{g}}$ is the generator matrix average ratio of ones per column.

$E_{o p}^{G}$ and $E_{o p}^{R}$ are the average energy per gate operation and register access, respectively.

The energy consumption for the RS code is defined as below:

$$
E_{R S}(n, k)=\left(E_{R S}^{e n}+\frac{E_{R S}^{d e c}}{\left(1-P_{b}\right)^{L+h s}}\right)+\left\lceil\frac{B a}{n_{c}(n-2 t) m}\right\rceil \times\left[n_{c}(n-2 t) m+\left\lceil\frac{h}{(n-2 t) m}\right\rceil+(n-2 t) m\right]
$$

where $\mathrm{n}$ is the total number of code symbols in the encoded blocks, $\mathrm{L}$ is the is the payload size, $h s$ is the header size and $B$ is the transmitted file size. $E_{R S}^{e n}$ and $E_{R S}^{d e c}$ are the energy consumed to encode and decode RS code respectively.

$$
\begin{aligned}
& n_{c}=\frac{L}{k m}, 2 t=n-k, \text { and } m=\log _{2}(n+1) \\
& E_{R S}^{e n}=\frac{927 t+657}{n-2 t} \\
& E_{R S}^{d e c}=\frac{995 t+2697}{n-2 t}+\left[1-\left(1-P_{b}\right)^{n m}\right] \frac{104 t^{2}+247 t+11730}{n-2 t}
\end{aligned}
$$

The others energy Consumption can be computed as follow:

$$
E_{D A C}=\frac{4 F_{D} n_{d} F_{S}}{R_{i}}
$$




$$
\begin{aligned}
& E_{\mathrm{mod}}=\frac{8 V_{c c} V_{p p}}{R_{T} R_{i}} \\
& P_{C W}=\boldsymbol{I}_{i} \cdot 10^{L_{\mathrm{mod}} / 10}
\end{aligned}
$$

$F_{D}$ is the DAC figure of merit, $n_{d}$ is the resolution of the DAC, Fs is the sample frequency, $V_{c c}$ is the modulator driver supply voltage, $V_{p p}$ is the modulator peak to peak swing voltage, $\mathrm{R}_{\mathrm{T}}$ is the modulator and driver termination resistance, $I_{i}$ is the input launch power for the $i_{t h}$ path and $\mathrm{L}_{\text {mod }}$ is optical excess loss of MZM.

The receptor energy Consumption can be computed as:

$$
E_{R X i}=\frac{R_{i}}{\eta_{E O}}\left(E_{L O}+E_{O E}+E_{A D C}+E_{T I A}+E_{D S P}\right)
$$

and the calculation of each variable is defined as follow:

$$
\begin{aligned}
& E_{L O}=\frac{16 \Re V_{\text {bias }} P_{\text {rec }}}{\varepsilon F_{S}} \\
& E_{T I A}=\frac{1.88}{R_{i} C_{L D P C} \log _{2}(M)} \\
& E_{A D C}=\frac{4 F_{D} n_{A D C} F_{S}}{R_{i}} \\
& E_{D S P}=E_{C D}+E_{T R}+E_{P M D}+E_{C R}+E_{D E C}
\end{aligned}
$$

$\mathrm{E}_{\mathrm{LO}}$ is the local oscillator energy consumption, $\mathrm{E}_{\mathrm{OE}}$ is the optical electrical converter energy consumption, $\mathrm{E}_{\mathrm{TIA}}$ is the trans-impedance amplifier with automatic gain control (AGC) energy consumption, $\mathrm{E}_{\mathrm{ADC}}$ is the analog-to-digital converter energy consumption and $\mathrm{E}_{\mathrm{DSP}}$ is the DSP energy consumption.

$\Re$ is the photodiode responsivity, $\mathrm{V}_{\text {bias }}$ is the photodiode bias voltage, $\mathrm{P}_{\mathrm{rec}}$ is the received power, $\mathrm{C}_{\mathrm{LDPC}}$ is the code rate, $\mathrm{M}$ constellation size, $\mathrm{n}_{\mathrm{ADC}}$ is the resolution of the $\mathrm{ADC}, \mathrm{E}_{\mathrm{CD}}$ is the energy consumption for $\mathrm{CD}$ compensation, $\mathrm{E}_{\mathrm{TR}}$ is the energy consumption for $\mathrm{TR}, \mathrm{E}_{\mathrm{PMD}}$ is the energy consumption for PMD compensation, $\mathrm{E}_{\mathrm{CR}}$ is the energy consumption for $\mathrm{CR}$ and $\mathrm{E}_{\mathrm{DEC}}$ is the energy consumption for decoding. 


\section{APPENDIX B: OPTICAL FIBER LINK PARAMETERS.}

\begin{tabular}{lcc}
\hline Variables & Value & Ref \\
\hline D - Dispersion parameter & $16.5(\mathrm{ps} /(\mathrm{nm} \mathrm{km}))$ & {$[24]$} \\
$\beta$ - Group velocity dispersion & $20.7(\mathrm{ps} / \mathrm{km})$ & {$[24]$} \\
$\lambda$ - Wavelength & $1550(\mathrm{~nm})$ & {$[24]$} \\
$\Delta \lambda$ - Channel spacing & $0.1(\mathrm{~nm})$ & {$[24]$} \\
$\gamma_{\mathrm{N}}$ - Fiber nonlinearity coefficient & $1.3(1 /(\mathrm{W} \mathrm{km}))$ & {$[24]$} \\
$\alpha$ - Fiber loss coefficient & $0.2(\mathrm{~dB} / \mathrm{km})$ & {$[24]$} \\
$L_{S}$ - Span length & $80(\mathrm{~km})$ & {$[25]$} \\
$h$ - Planck constant & $6,63 \times 10^{34}(\mathrm{~J} / \mathrm{Hz})$ & {$[25]$} \\
$\mathrm{v}-$ Light frequency & $1931(\mathrm{THz})$ & {$[25]$} \\
$\mathrm{N}_{\text {node, }}$ - BV-OXC Noise power penalty & $1 \mathrm{~dB}$ & {$[25]$} \\
$\mathrm{N}_{\mathrm{c}}$ - Number of channels & 100 & {$[26]$} \\
Pe - Symbol-error prob. (without FEC) & $10^{5}$ & \\
\hline
\end{tabular}

APPENDIX C: POWER CONSUMPTION VARIABLES

\begin{tabular}{|c|c|c|}
\hline Variable & Adopted Value & Ref \\
\hline$\eta_{\mathrm{EO}}-$ Power conversion efficiency & $1 \%$ & [6] \\
\hline$\eta_{T}-$ Transponder efficiency & 1.5 (W/Gbps) & [27] \\
\hline$E_{o p}^{G}$ - Energy per gate operation & $0.69 \mathrm{ptV}^{2}(\mathrm{fJ})$ & {$[18]$} \\
\hline$E_{o p}^{R}$ - Energy per register access & $3.43 \mathrm{ptV}^{2}(\mathrm{fJ})$ & {$[18]$} \\
\hline B - Transmitted file size & $100(\mathrm{~GB})$ & {$[28]$} \\
\hline$N_{f}-$ Number of frames & 107 (frames) & [28] \\
\hline$h_{s}-$ Header size & $64(B)$ & {$[28]$} \\
\hline$\varepsilon-$ Spectral efficiency & $1,2,3,4,5,6(\mathrm{~b} / \mathrm{s} / \mathrm{Hz})$ & {$[14]$} \\
\hline $\mathrm{E}_{\mathrm{PS}}-$ Parallel/serial conversion & $4.0 / \varepsilon . \mathrm{F}_{\mathrm{S}}(\mathrm{J} / \mathrm{bit})$ & {$[6]$} \\
\hline$F_{D}$ - DAC figure of merit & $1.56 \times 10^{12}(\mathrm{~J} /$ conv $)$ & {$[29]$} \\
\hline$n_{d}-$ DAC resolution & $4,6,8$ (bits) & [29] \\
\hline $\mathrm{F}_{\mathrm{S}}$ - Sampling frequency & $2 \mathrm{R} / \varepsilon(\mathrm{Hz})$ & [29] \\
\hline Vcc - Modulator driver supply voltage & $5.2(\mathrm{~V})$ & {$[18]$} \\
\hline Vpp - Modulator peak swing voltage & $8(\mathrm{~V})$ & {$[18]$} \\
\hline $\mathrm{R}_{\mathrm{T}}$ - Modulator termination resistance & 50() & {$[6]$} \\
\hline
\end{tabular}




\begin{tabular}{lcc}
\hline $\mathrm{L}_{\text {mod }}$ Optical excess loss & $2.5(\mathrm{~dB})$ & {$[6]$} \\
$\Re$ - Photodiode responsivity & $0.9(\mathrm{~A} / \mathrm{W})$ & {$[6]$} \\
$\mathrm{V}_{\text {bias }}$ - Photodiode bias voltage & $3.3(\mathrm{~V})$ & {$[6]$} \\
$\mathrm{C}_{\mathrm{LDPC}}$ - Code rate & 0.83 & {$[30]$} \\
$\mathrm{n}_{\mathrm{ADC}}-\mathrm{DAC}$ resolution & $8(\mathrm{bits})$ & {$[30]$} \\
$\mathrm{E}_{\mathrm{LO}}-$ Local oscillator consumption & $5 / \varepsilon . \mathrm{F}_{\mathrm{S}}(\mathrm{pJ} / \mathrm{bit})$ & {$[18]$} \\
$\mathrm{E}_{\mathrm{CD}}-\mathrm{CD}$ compensation consumption & $324 / \varepsilon . \mathrm{F}_{\mathrm{S}}(\mathrm{pJ} / \mathrm{bit})$ & {$[18]$} \\
$\mathrm{E}_{\mathrm{TR}}-\mathrm{TR}$ consumption & $104 / \varepsilon . \mathrm{F}_{\mathrm{S}}(\mathrm{pJ} / \mathrm{bit})$ & {$[18]$} \\
$\mathrm{E}_{\mathrm{PMD}}-\mathrm{PMD}$ compensation consumption & $246 / \varepsilon . \mathrm{FS}(\mathrm{pJ} / \mathrm{bit})$ & {$[18]$} \\
$\mathrm{E}_{\mathrm{CR}}-$ Carrier recovery consumption & $56 / \varepsilon . \mathrm{F}_{\mathrm{S}}(\mathrm{pJ} / \mathrm{bit})$ & {$[18]$} \\
$\mathrm{P}_{\mathrm{Sat}}$ EDFA power saturation & $16(\mathrm{dBm})$ & {$[31]$} \\
$\mathrm{G}_{0}-\mathrm{Spectral}$ EDFA gain & 15 & {$[21]$} \\
$\eta_{\mathrm{EPCE}}-$ EDFA conversion efficiency & $1 \%$ & {$[6]$} \\
\hline
\end{tabular}

\section{ACKNOWLEDGMENT}

This work is supported by CAPES, CNPQ and FAPEMIG.

\section{REFERENCES}

[1] Lange, C. et al., Energy consumption of telecommunication networks. In: IEEE. Optical Communication, ECOC'09. 35th European Conference on. [S.1.], p1-2, 2009.

[2] Koutitas, G.; Demesticha S, P., A review of energy efficiency in telecommunication networks. Telfor journal, v. 2, n. 1, p. 2-7, 2010.

[3] Rouzic, E. L., Network evolution and the impact in core networks. In: IEEE. Optical Communication (ECOC), 36th European Conference and Exhibition on. [S.1.], p. 1-8, 2010.

[4] PickaveT, M.; Tucker, R., Network solutions to reduce the energy footprint of ict. In: European conference on optical communications (ECOC 2008), Brussels, Belgium. [S.1.: s.n.], p. 21-25, 2008.

[5] Ricciardi, S. et al., An energy-aware dynamic rwa framework for next-generation wavelength-routed networks. Computer Networks, Elsevier, v. 56, n. 10, p. 2420-2442, 2012

[6] Tucker, R. S., Green optical communications - part i: Energy limitations in transport. IEEE Journal of selected topics in quantum electronics, IEEE, v. 17, n. 2, p. 245-260, 2011.

[7] Lange, C. et al., Energy consumption of telecommunication networks and related improvement options. IEEE Journal of selected topics in quantum electronics, IEEE, v. 17, n. 2, p. 285-295, 2011.

[8] Maheswaran, D. and K. K. J. Kailas, Energy efficiency in electrical systems", IEEE International Conference on Power Electronics, Drives and Energy Systems, Bengaluru, India, Dec. 16-19, 2012

Brazilian Microwave and Optoelectronics Society-SBMO received 09 Oct 2017; for review 13 Oct 2017; accepted 01 Feb 2018 
[9] Rodney, T., Green optical communications Part I: Energy limitations in transport”, IEEE Journal of selected topics in quantum electronics, v.17, n. 2, p. 245-260, 2011.

[10] Gerstel, O. et al., Elastic optical networking: A new dawn for the optical layer? IEEE Communications Magazine, IEEE, v. 50, n. 2, 2012

[11] Jijun, Z. et al, Distance-adaptive routing and spectrum assignment in OFDM-based flexible transparent optical networks", Photonic Network Communications 27.3, p. 119-127, 2014.

[12] Jinno, M. H.; Takara, B. Kozicki, Y. Tsukishima, Y. Sone, and S. Matsuoka, Spectrum-efficient and scalable elastic optical path network: Architecture, benefits, and enabling technologies”, IEEE Commun. Mag., vol. 47, no. 11, pp. 6673, Nov. 2009

[13] Christodoulopoulos, K. I. T, and E. Varvarigos, Elastic bandwidth allocation in flexible OFDM-based optical networks, IEEE J. Lightw. Technol., vol. 29, no. 9, pp. 13541366, May 2011.

[14] Zhao, J. et al, Distance-adaptive routing and spectrum assignment in ofdm-based flexible transparent optical networks Photonic Network Communications, Springer, v. 27, n. 3, p. 119-127, 2014.

[15] Wang, Y.; Cao, X.; Pan, Y. A study of the routing and spectrum allocation in spectrum sliced elastic optical path networks. In: IEEE. INFOCOM, 2011 Proceedings IEEE. [S.1.], p. 1503-1511, 2011.

[16] Li Y. et al., Adaptive FEC-based lightpath routing and wavelength assignment in WDM optical networks", Optical Switching and Networking 14 , P241-249, 2014.

[17] Sab, O. A.; Lemaire, V. Block turbo code performances for long-haul dwdm optical transmission systems. In: IEEE. Optical Fiber Communication Conference, 2000. [S.1.], v. 3, p. 280-282, 2000.

[18] Pillai B. S. G. et Al, End-to-end energy modeling and analysis of long-haul coherent transmission systems, Journal of Lightwave Technology, VOL. 32, NO. 18, SEPTEMBER 15, 2014

[19] Klekamp, A.; Dischler, R.; Buchali, F., Transmission reach of optical-ofdm superchannels with 10-600 gb/s for transparent bit-rate adaptive networks. In: OPTICAL SOCIETY OF AMERICA. European Conference and Exposition on Optical Communications. [S.1.], p. Tu-3, 2011.

[20] Jin Yen, Finding the k-shortest loopless paths in a network”, Management Science 17.11, p. 712-716, 1971.

[21] Durand F. and A. Taufik, Energy efficiency analysis in adaptive FEC based lightpath elastic optical networks, Journal of Circuits, Systems and Computers, v. 24, n. 09, p. 1550133, 2015.

[22] Bhide, N. M.; Sivalingam, K. M.; Fabryasztalos, T.., Routing mechanisms employing adaptive weight functions for shortest path routing in optical WDM networks. Photonic Network Communications, v. 3, n. 3, p. 227-236, 2001.

[23] Wen, Bo; Shenai, R.; Sivalingam, K., Routing, wavelength and time-slot-assignment algorithms for wavelengthrouted optical WDM/TDM networks. Journal of Lightwave Technology, v. 23, n. 9, p. 2598, 2005.

[24] Poggiolini, Pierluigi et al. The GN-model of fiber non-linear propagation and its applications. Journal of lightwave technology, v. 32, n. 4, p. 694-721, 2014.

[25] Gao, G. J. Zhang, L. Wang, W. Gu and Y. Ji, Influence of physical layer configuration on performance of elastic optical OFDM networks, IEEE Comm. Lett. 18 (2014) 672-675.

[26] Barros, D. and J. Kahn, Comparison of orthogonal frequency-division multiplexing and on-off keying in amplified direct-detection single-mode fiber systems, J. Lightwave Technol. 28 (2010) 1811-1820.

[27] Heddeghem, H. et al., Power consumption modeling in optical multilayer networks, Photon Netw. Commun. 24 (2012) 86-102.

[28] Guan, K. B. S. G. Pillai, A. Vishwanath, D. C. Kilper and J. Llorca, The impact of error control on energy-efficient reliable data transfers over optical networks, Proc. Int. Conf. Communications (ICC'13), Budapest, June 2013, doi: 10.1109/ICC.2013.6655200

[29] Walden, R., Analog-to-digital converter survey and analysis, IEEE J. Sel. Areas Commun. 17 (1999) 539-550.

[30] Kuschnerov, M. T. Bex and P. Kainzmaier, Energy etcient digital signal processing, Proc. OFC 2014, March 2014, Paper Th3E.7. 
[31] Pereira, H. D. Chaves, C. Bastos-Filho and J. Martins-Filho, OSNR model to consider physical layer impairments in transparent optical networks, Photon. Netw. Commun. 18 137-149, 2009. 